IAC-10.C4.6.8

\title{
HYBRID SOLAR SAIL AND SEP PROPULSION FOR NOVEL EARTH OBSERVATION MISSIONS
}

\author{
Matteo Ceriotti \\ Advanced Space Concepts Laboratory, University of Strathclyde, Glasgow, United Kingdom \\ $\underline{\text { matteo.ceriotti@strath.ac.uk }}$ \\ Colin R. McInnes \\ Advanced Space Concepts Laboratory, University of Strathclyde, Glasgow, United Kingdom \\ colin.mcinnes@strath.ac.uk
}

In this paper we propose a pole-sitter spacecraft hybridising solar electric propulsion (SEP) and solar sailing. The intriguing concept of a hybrid propulsion spacecraft is attractive: by combining the two forms of propulsion, the drawbacks of the two systems cancel each other, potentially enabling propellant mass saving, increased reliability, versatility and lifetime over the two independent systems. This almost completely unexplored concept will be applied to the continuous monitoring of the Earth's polar regions through a pole-sitter, i.e. a spacecraft that is stationary above one pole of the Earth. The continuous, hemispherical, real-time view of the pole will enable a wide range of new applications for Earth observation and telecommunications. In this paper, families of 1-year-periodic, minimum-propellant orbits are found, for different values of the sail lightness number and distance from the pole. The optimal control problem is solved using a pseudo-spectral method. The process gives a reference control to maintain these orbits. In addition, for stability issues, a feedback control is designed to guarantee station-keeping in the presence of injection errors, sail degradation and temporary SEP failure. Results show that propellant mass can be saved by using a medium-sized solar sail. Finally, it is shown that the feedback control is able to maintain the spacecraft on-track with only minimal additional effort from the SEP thruster.

\section{INTRODUCTION}

Geostationary spacecraft provide a continuous hemispherical view of equatorial and medium-latitude zones, but unfortunately these platforms cannot cover high-latitude regions. Conversely, a pole-sitter is a spacecraft that is constantly above one of the Earth's poles, i.e. lying on the Earth's polar axis [1]. This type of mission would provide a continuous, hemispherical, real-time view of the pole, and will enable a wide range of new applications in climate science and telecommunications.

Traditionally, polar observation is performed with one spacecraft or a constellation of spacecraft in highlyinclined low or medium Earth orbits [2]; however, the observation is not continuous, but it relies on the passage of one spacecraft above regions of interest. Therefore, the temporal coverage of the entire polar region can be quite poor, and furthermore different areas are imaged at different times, hence missing the possibility to have a real-time complete view of the pole.

The hemispheric view of the pole is currently reconstructed through a composite image that is made of several images taken at different times. The main science applications for polar image composites are the generation of atmospheric motion vectors (AMV) and the identification of storm systems. According to Lazzara [3], these two applications among others would benefit from a true pole-sitter spacecraft. For example, both at the North Pole and South Pole, there is an interval of latitudes in which the AMV are not available, due to a gap between geostationary and polar orbiting satellites. Citing Lazzara, "the possibility of seeing the deep polar regions with dramatically increased temporal resolution, and even the opportunity for focused observations on a specific geographic region, with potential high spatial and temporal resolution, are capabilities that would improve the short term forecasting and understanding of atmospheric phenomena that occur in these portions of the world".

Another significant benefit to the polar regions from a pole-sitter platform will be for communications. It is well known that line-of-sight telecommunications to conventional spacecraft in geostationary orbits is not possible at high latitudes and polar regions. While the distance of the spacecraft from the Earth could preclude high-bandwidth telecommunications, a pole-sitter will be able to always see the South Pole, providing a continuous flow of data with scientific South Pole stations. The same spacecraft could be accomplish both observation and telecommunication tasks over the polar regions. Furthermore, telecommunication with polar regions will be a key issue in future as changes to the arctic ice pack opens navigation channels for shipping. Such developments will necessitate real-time polar imaging for route planning.

Here we propose to hybridise solar electric propulsion (SEP) with solar sailing, in order to get 
benefit from combining the two propulsion systems on the same spacecraft.

Solar electric propulsion (SEP) is a mature technology that provides a spacecraft with a relatively low thrust (of the order of a fraction of a Newton [4]). Nevertheless, despite their high efficiency, the thrusting time and hence the mission duration is always limited by the mass of propellant on-board.

In contrast, solar sailing [5] is a propellant-less spacecraft propulsion system: it exploits the solar radiation pressure due to solar photons impinging on a large, highly reflecting surface (the sail) to generate thrust. Despite the original idea of solar sailing being rather old [6], only very recently a spacecraft successfully deployed a solar sail: JAXA's IKAROS [7].

Due to the interesting potential of enabling missions that are not constrained by propellant mass, studies on potential solar sail missions have been undertaken, while others are still ongoing $[8,9]$. Solar sails seem to be suitable for potentially long duration missions that require a small, but continuous, thrust. They have been proposed for interplanetary transfers [10], or to generate artificial equilibrium points [11], for example in the proximity of the Lagrange points of the Sun-Earth [12] or Sun-Moon [13] system.

The idea of hybridise solar sail propulsion and solar electric propulsion (SEP) on the same spacecraft was proposed in 2002 [14] and is almost completely unexplored. Current research ranges from artificial equilibria in the Sun-Earth system for Earth observation [15] to interplanetary transfers [16], to displaced periodic orbits in the Earth-Moon system [17]. In addition, IKAROS is exploiting hybrid propulsion [7].

The reason for this interest is due to the fact that in the hybrid system, at the cost of increased spacecraft complexity, the two propulsion systems complement each other, cancelling their reciprocal disadvantages and limitations. In principle, SEP can provide the missing acceleration component towards the Sun, that the sail cannot generate. Similarly, the hybrid spacecraft can be seen as an SEP spacecraft, in which an auxiliary solar sail provides part of the acceleration, enabling saving of propellant mass, and lower demand on the electric thruster, possibly with some intervals in which it could be switched off. Moreover, the reliability of the system is increased: the solar sail is only providing part of the necessary acceleration, and hence it can be smaller than what would be required for a pure sail spacecraft; also, the sail deployment is not a single-point failure, as the SEP could serve as a propulsion backup. In this case, a degraded, possibly shorter mission can be foreseen. In this sense, the hybrid spacecraft can be seen as a way to gradually introduce solar sails for space applications, and hence to reduce the advancement degree of difficulty $\left(\mathrm{AD}^{2}\right)[18]$ in the TRL scale.
In this work we propose hybrid propulsion for the pole-sitter spacecraft, thus extending the pure SEP spacecraft proposed by Driver [1] and the hybrid spacecraft stationary above the Lagrangian point $L_{1}$ proposed by Baig and McInnes [15], providing a practical realisation of the solar sail pole-sitter orbits by Forward [19].

Previous work by the authors focused on the optimisation procedure, including a shape-based approach to find first-guess solutions [20], and a comparison between pure SEP and hybrid sails in terms of spacecraft mass [21]. In this paper we present the design and control of optimal orbits for a pole-sitter mission. After explaining the dynamics that will be used, we will present the optimal control problem that is solved to obtain minimum propellant consumption orbits. After a comparison between the hybrid sail and pure SEP performance, a feedback control system will be designed to keep the spacecraft on track when subject to injection error, sail degradation, or temporary SEP failure.

\section{EQUATIONS OF MOTION}

We consider the circular restricted three-body problem (CR3BP), that describes the motion of a negligible mass (the spacecraft) under the gravitational attraction of two masses (the primaries, Sun and Earth in this case), that rotate of circular motion around their common centre of mass. We use a synodic reference frame centred at the centre of mass, and having the $\hat{\mathbf{x}}$ axis collinear with the two primaries, pointing towards the Earth, the $\hat{\mathbf{z}}$ axis is aligned with the angular velocity of the primaries $\boldsymbol{\omega}=\omega \hat{\mathbf{z}}$ and the $\hat{\mathbf{y}}$ axis completes the right-hand system (Fig. 1).

The dynamics of a spacecraft at position $\mathbf{r}$ is governed by:

$$
\ddot{\mathbf{r}}+2 \boldsymbol{\omega} \times \dot{\mathbf{r}}-\nabla U=\mathbf{a}_{s}+\mathbf{a}_{T}
$$

where $\mathbf{r}$ is the position vector and the effective potential, which takes into account gravitational attraction and centrifugal acceleration, is:

$$
U=\frac{1}{2}\left(r_{x}^{2}+r_{y}^{2}\right)+\frac{1-\mu}{r_{1}}+\frac{\mu}{r_{2}}
$$

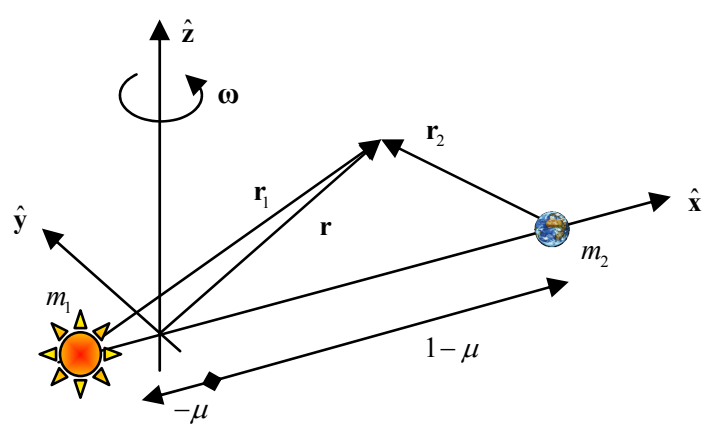

Fig. 1: The restricted three-body problem. 


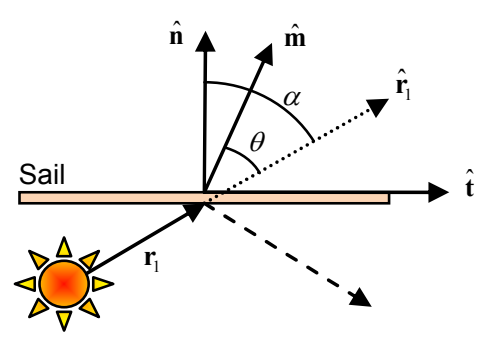

a)

Fig. 2. (a) Definition of the cone and centre-line angles (plane of the figure is perpendicular to the sail, containing the Sun vector $\mathbf{r}_{1}$ ). (b) Solar sail cone and clock angles.

The system is normalised such that $\omega=1$, $m_{1}+m_{2}=1$, and the unit of distance is the separation of the two primaries. With these assumptions, the position along the $\hat{\mathbf{x}}$-axis of $m_{1}$ is $-\mu$, and the position of $m_{2}$ is $1-\mu$, and $\mu=3.0404 \cdot 10^{-6}$.

In addition, $\mathbf{a}_{s}$ and $\mathbf{a}_{T}$ are the accelerations provided by the solar sail and the SEP thruster, respectively. The former is expressed as [5]:

$$
\mathbf{a}_{s}=\frac{1}{2} \beta_{0} \frac{m_{0}}{m} \frac{1-\mu}{r_{1}^{2}}[g \cos \alpha \hat{\mathbf{n}}+h \sin \alpha \hat{\mathbf{t}}] \cos \alpha
$$

Here $\hat{\mathbf{n}}$ is the component normal to the non-ideal sail and parallel $\hat{\mathbf{t}}$ to it, in the plane of the Sun vector $\mathbf{r}_{1} . \beta_{0}$ is the lightness number at the beginning of the mission, $\beta_{0}=\sigma^{*} A / m_{0}$ : values of $\beta_{0}$, ranging from 0 (no sail, hence pure SEP spacecraft) to 0.05 can be assumed for near- to mid-term technology [22]. $m_{0}$ and $m$ are the spacecraft mass at the beginning of the mission and at any given time, respectively. Note that, in the hybrid case, the spacecraft mass varies in general, due to the SEP propellant consumption, and so does the acceleration from the sail. $\sigma^{*} \cong 1.53 \cdot 10^{-3} \mathrm{~kg} / \mathrm{m}^{2}$ is the critical sail loading for the Sun.

The sail acceleration is controlled through the spacecraft attitude: the vector $\hat{\mathbf{n}}$ can be described using the cone angle $\alpha$ (angle between $\hat{\mathbf{n}}$ and $\hat{\mathbf{r}}_{1}$, see Fig. 2a) and the clock angle $\delta$ (angle measured around $\hat{\mathbf{r}}_{1}$, starting from the vertical plane, of the component of $\hat{\mathbf{n}}$ perpendicular to $\hat{\mathbf{r}}_{1}$, see Fig. 2b). In the hybrid spacecraft, thin film solar cells (TFSC) cover an area $A_{T F}=0.05 \mathrm{~A}$ on the sail, and are used to power the SEP thruster (see Fig. 3 for a potential configuration). The area ratio is a conservative estimation based on previous studies [15]. The actual direction of the sail acceleration $\hat{\mathbf{m}}$ is related to $\hat{\mathbf{n}}$ through the coefficients $g$ and $h$ [15], that can be computed as a function of the reflectivity of the sail, $\tilde{r}_{s}=0.9$, and of the thin film $\tilde{r}_{T F}=0.4$ [14]:

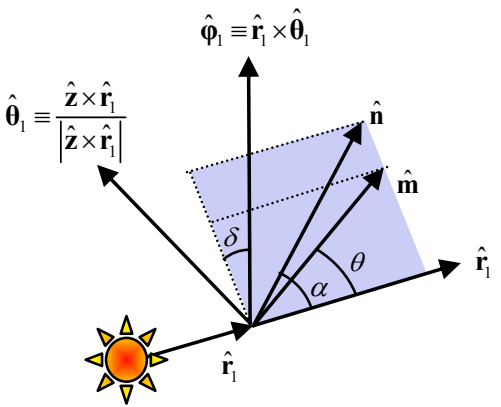

b)

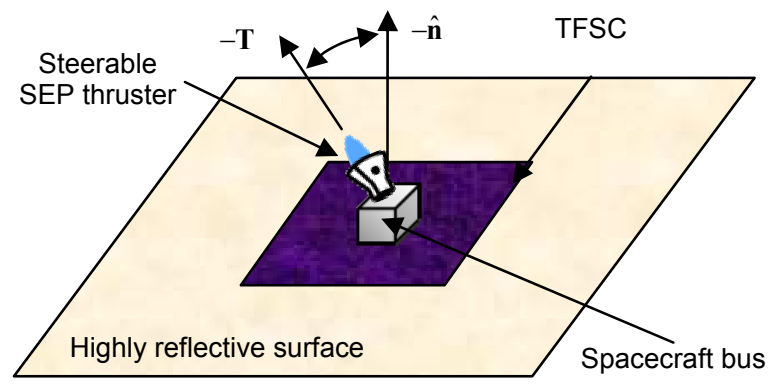

Fig. 3: Configuration of the hybrid sail/SEP spacecraft, with steerable thrust.

$$
\begin{aligned}
& g=1+\tilde{r}_{s}+\frac{A_{T F}}{A}\left(\tilde{r}_{T F}-\tilde{r}_{s}\right) \\
& h=1-\tilde{r}_{s}-\frac{A_{T F}}{A}\left(\tilde{r}_{T F}-\tilde{r}_{s}\right)
\end{aligned}
$$

The SEP thruster is assumed to be variable-thrust and mounted on a gimbal, such that the thrust vector $\mathbf{T}$ is completely controllable (refer again to Fig. 3). The propellant mass flow $\dot{m}$ is related to the thrust through the Newton's law and the conservation of mass:

$$
\dot{m}=T / v_{e}
$$

where the exhaust gas velocity is $v_{e}=I_{s p} g_{0} . I_{s p}$ is the specific impulse in seconds and $g_{0}=9.81 \mathrm{~m} / \mathrm{s}^{2}$.

\section{POLE-SITTER MISSION}

A pole-sitter spacecraft, during the operational phase of its mission, is constantly aligned with the polar axis of the Earth. If we neglect precession of the equinoxes and nutation (which are long-term, and thus irrelevant in this analysis), the polar axis of the Earth does not change its direction while the Earth is orbiting the Sun. Therefore, in the synodic reference frame, the same axis rotates with a motion of apparent precession. Its angular velocity is the opposite of that of the primaries, or $-\boldsymbol{\omega}$. Therefore the polar axis spans a full conical surface every year (see Fig. 4). The cone half angle is the tilt of the axis relative to the ecliptic, i.e. $\delta_{e q}=23.5 \mathrm{deg}$. 


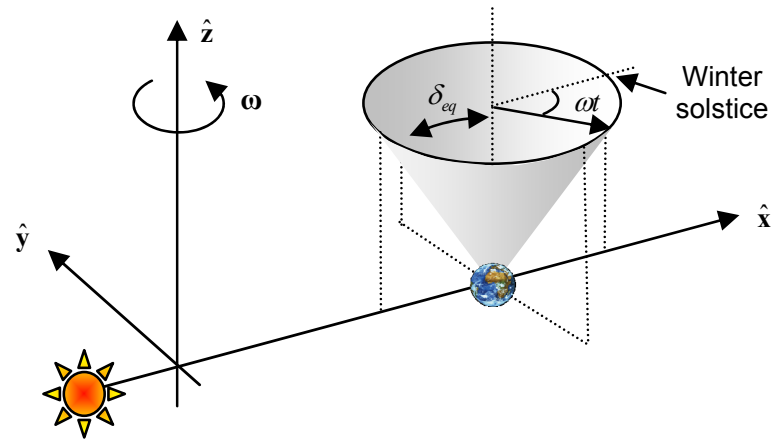

Fig. 4: Apparent precession of the Earth's polar axis due to rotation of reference frame.

Consequently, the pole-sitter shall follow the Earth's polar axis, and describe a 1-year-periodic orbit which can be described by:

$$
\mathbf{r}(t)=\left[\begin{array}{c}
d(t) \sin \delta_{e q} \cos \omega t+(1-\mu) \\
-d(t) \sin \delta_{e q} \sin \omega t \\
d(t) \cos \delta_{e q}
\end{array}\right]
$$

where $d(t)$ is the distance from the centre of the Earth, and is a continuous function of time. The North Pole case is considered here.

In this work, we search for optimal periodic polesitter orbits, that minimise the SEP propellant consumption over a period (one year), while maintaining the pole-sitter condition (3) at each time during the mission.

\section{III.I. Optimal Control Problem}

Since the periodic orbit, i.e. the function $d(t)$, is not known a priori, an optimal control problem is solved to find it, together with the control history that guarantees minimal fuel consumption. The problem is the one of finding the state time history $\mathbf{x}(t) \in \mathbb{R}^{n_{x}}$ and control time history $\mathbf{u}(t) \in \mathbb{R}^{n_{u}}$, between initial and final time, $t_{0}$ and $t_{f}$, minimising the cost function:

$$
J=\varphi\left(\mathbf{x}_{0}, \mathbf{x}_{f}, t_{0}, t_{f}\right)+\int_{t_{0}}^{t_{f}} L\left(\mathbf{x}\left(t^{\prime}\right), \mathbf{u}\left(t^{\prime}\right), t^{\prime}\right) \mathrm{d} t^{\prime}
$$

subject to the dynamical constraints:

$$
\dot{\mathbf{x}}(t)=\mathbf{f}(\mathbf{x}(t), \mathbf{u}(t), t) \in \mathbb{R}^{n_{x}}
$$

state and control bounds:

$$
\begin{aligned}
& \mathbf{x}_{l} \leq \mathbf{x}(t) \leq \mathbf{x}_{u} \\
& \mathbf{u}_{l} \leq \mathbf{u}(t) \leq \mathbf{u}_{u}
\end{aligned}
$$

initial and final state bounds:

$$
\begin{aligned}
& \mathbf{x}_{0, l} \leq \mathbf{x}\left(t_{0}\right) \leq \mathbf{x}_{0, u} \\
& \mathbf{u}_{0, l} \leq \mathbf{u}\left(t_{0}\right) \leq \mathbf{u}_{0, u} \\
& \mathbf{x}_{f, l} \leq \mathbf{x}\left(t_{f}\right) \leq \mathbf{x}_{f, u} \\
& \mathbf{u}_{f, l} \leq \mathbf{u}\left(t_{f}\right) \leq \mathbf{u}_{f, u}
\end{aligned}
$$

and general non-linear path constraints:

$$
\mathbf{c}(\mathbf{x}, \mathbf{u}, t) \leq 0
$$

For this specific case, the time span in normalised units is $t_{0}=0, t_{f}=2 \pi$. The state vector is composed of the position, velocity and mass $\mathbf{x}=\left[\begin{array}{lll}\mathbf{r} & \mathbf{v} & m\end{array}\right]^{T}$ of the spacecraft, which leads to the following dynamical constraints:

$$
\dot{\mathbf{x}}=\left[\begin{array}{c}
\mathbf{v} \\
-\nabla U-2 \boldsymbol{\omega} \times \mathbf{v}+\mathbf{a}_{s}+\mathbf{T} / m \\
-T / v_{e}
\end{array}\right]
$$

The controls (sail normal $\hat{\mathbf{n}}$ and thrust vector $\mathbf{T}$ ) are expressed in Cartesian components in the reference frame in Fig. $2 b$, instead of angles and magnitude, to avoid the ambiguity related to the use of angular variables:

$$
\mathbf{u}=\left[\begin{array}{llllll}
n_{r_{1}} & n_{\theta_{1}} & n_{\varphi_{1}} & T_{r_{1}} & T_{\theta_{1}} & T_{\varphi_{1}}
\end{array}\right]^{T}
$$

Since the sail normal $\hat{\mathbf{n}}$ is a unit vector, a path constraint shall be added to guarantee the uniqueness of the solution:

$$
\sqrt{n_{r_{1}}^{2}+n_{\theta_{1}}^{2}+n_{\varphi_{1}}^{2}}=1
$$

The pole-sitter constraint in Eq. (3) is enforced through the path constraints:

$$
\begin{aligned}
& \operatorname{atan} 2\left(-r_{2, y}, r_{2, x}\right)-\omega t=0 \\
& \sqrt{r_{2, x}^{2}+r_{2, y}^{2}}-r_{2, z} \tan \left(\delta_{e q}\right)=0
\end{aligned}
$$

We assume that the spacecraft is injected in the polesitter orbit at the winter solstice, at some point in the $\mathbf{x}-\mathbf{z}$ plane. To guarantee that the orbit is periodic and symmetric with respect to the same plane, the initial velocity can only have a positive $\mathbf{y}$ component. Additionally it is enforced that the position and velocity at time $t_{f}$ match those (partially to be determined) at time $t_{0}$.

The objective is to maximise the final mass of the spacecraft, i.e. minimise the fuel consumption, after one period:

$$
J=-m_{f}=-m\left(t_{f}\right)
$$

To solve this optimal control problem, the tool PSOPT was used [23]. PSOPT implements a direct pseudo-spectral method to solve the optimal control problem. By discretising the time interval into a finite number of nodes, the infinite dimensional optimal control problem is transformed into a finite dimension non-linear programming (NLP) problem. Pseudospectral methods use Legendre or Chebyshev polynomials to interpolate the time dependent variables at the nodes. The advantage of using pseudo-spectral methods is that the derivatives of the state functions at the nodes are computed by matrix multiplication only, and that the integral in the cost function is approximated 


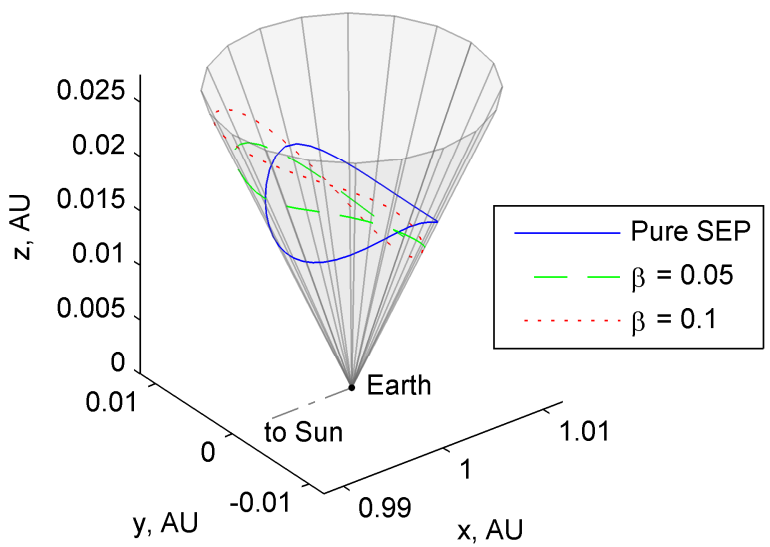

Fig. 5: Optimal hybrid pole-sitter orbits for three different values of $\beta_{0}$.

using well known Gauss quadrature rules. We refer to $[24,25]$ for a detailed description of pseudo-spectral methods.

The optimisation process is initialised with a firstguess solution. The generation of this sub-optimal trajectory, and corresponding control history, is the subject of previous work [20]. Here we provide a brief outline of the procedure. The first guess is generated by using a shape-based approach, in which a specific orbit for the spacecraft and initial mass $m_{0}$ are assigned, and then the controls that enable that orbit are obtained from the equations of motion, with an iterative process. The orbit is discretised into a finite number of points in time. At each point, the sail cone and clock angles are computed numerically, minimising the magnitude of the SEP acceleration. Once $\mathbf{a}_{s}$ is known, $\mathbf{a}_{T}$ can be computed by differencing. Assuming that the thrust remains constant from one point to the next along the orbit, Eq. (2) can be integrated to find the mass change. With this new value of mass, the procedure iterates on to the next point on the orbit.

\section{III.II. Solutions}

Optimal orbits are sought for three different spacecraft: a pure SEP (no solar sail, $\beta_{0}=0$ ) and two different hybrid spacecraft, with increasing size of the sail $\left(\beta_{0}=0.05,0.1\right)$. The initial mass is $m_{0}=1000 \mathrm{~kg}$ and $I_{s p}=3000 \mathrm{~s}$ (based on current ion engine technology (existing NSTAR/DS1 [26]).

Each case leads to a different optimal orbit (Fig. 5). The pure SEP spacecraft optimal orbit is symmetric with respect to summer and winter, while if a solar sail is added, the spacecraft goes farther from the Earth in summer and closer in winter. All the orbits are at about

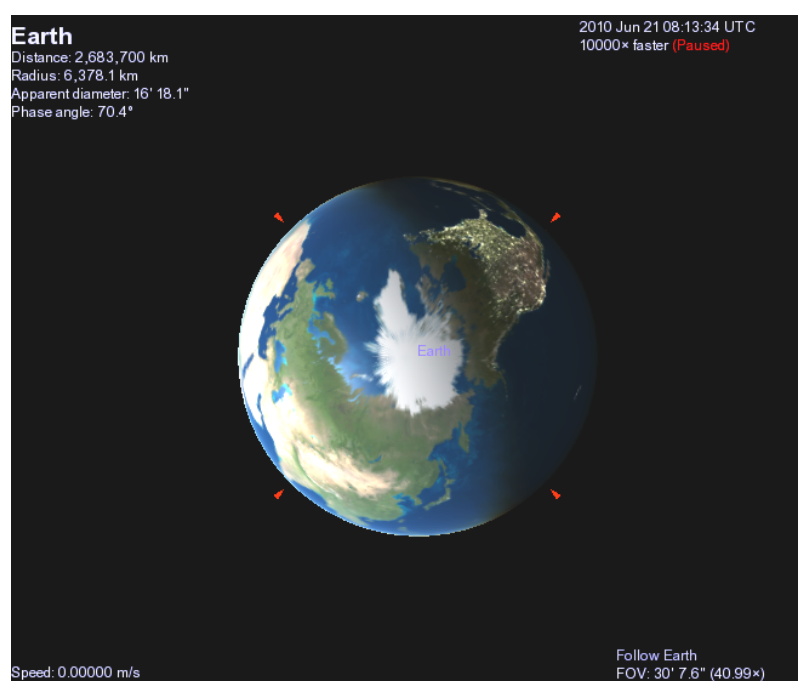

Fig. 6: The Earth as seen from the pole-sitter spacecraft at 0.018 AU using an instrument with 30 ' field of view.

Table 1: Optimal pole-sitter orbits for three different values of lightness number.

\begin{tabular}{ccccc}
\hline$\beta_{0}$ & $d_{\min }, \mathrm{AU}$ & $d_{\max }, \mathrm{AU}$ & $m_{f}, \mathrm{~kg}$ & $T_{\max }, \mathrm{N}$ \\
\hline 0.0 & 0.015675 & 0.020332 & 843.430417 & 0.180648 \\
0.05 & 0.013116 & 0.023422 & 901.896219 & 0.141085 \\
0.1 & 0.011896 & 0.028363 & 925.192867 & 0.134256 \\
\hline
\end{tabular}

0.018 AU (or about 2.7 millions of $\mathrm{km}$ ) from the Earth's centre, as can be noted in Table 1. Fig. 6 shows the view of the Earth from this distance. Note that the Lagrangian point $L_{1}$ of the Earth-Sun system is about 1.5 million $\mathrm{km}$ from the Earth. Fig. 5 shows the three orbits: in the same plot, the cone described by the Earth's polar axis is also superimposed. Table 1 also highlights the propellant mass saving that a hybrid solar sail offers with respect to pure SEP. Also, the presence of the sail allows us to reduce the maximum thrust needed, thus allowing the use of a smaller engine.

However, it shall be noted that these results assume a spacecraft with the same initial mass, both in the case of SEP only or hybrid propulsion. If a sail is present, then the dry mass of the spacecraft shall be higher or the payload mass reduced. A complete investigation of the mass budget of the hybrid spacecraft, including a discussion on the benefit of the hybrid propulsion in terms of mass saving, is presented in [21].

It was stated that the thrust vector is assumed completely controllable, from a trajectory design point of view. However this cannot be achieved in practice. Although a gimbal system can be used, the thruster cannot eject propellant in the direction of the solar sail, in the case of the hybrid system. Also it is difficult to engineer a thruster that could rotate 180 degrees. Therefore, we analysed the steering angle needed for the 


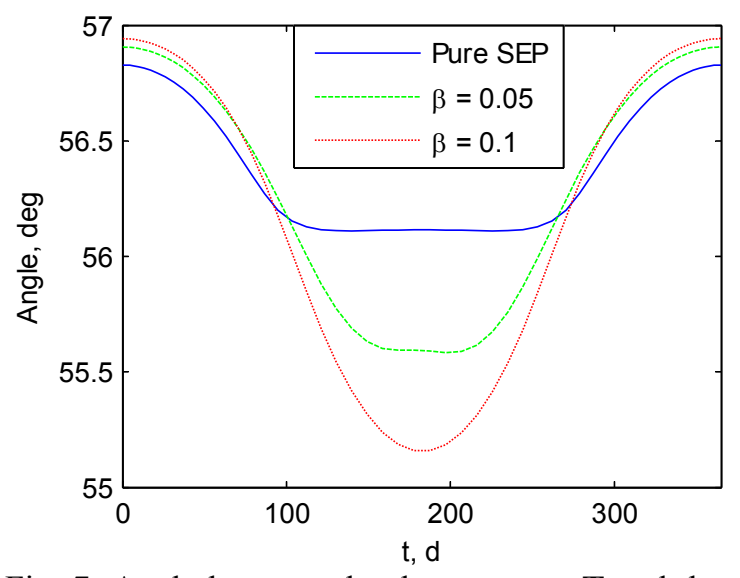

Fig. 7: Angle between the thrust vector $\mathbf{T}$ and the solar sail normal $\mathbf{n}$, during the three orbits in Fig. 5.

thruster, with respect to the main spacecraft bus (and the sail. Fig. 7 shows the evolution during one orbit of the angle between the sail normal and the thrust vector: this is the angle represented in Fig. 3. Two important considerations can be noted: the first is that the angle variation is limited to about $2 \mathrm{deg}$, for all the three cases presented. This implies very little movement of the thruster, and possibly a lightweight gimbal mechanism. The second is that the angle is around $56 \mathrm{deg}$, and therefore the thruster does not thrust towards the sail, during the whole orbit.

Additional families of orbits can be found by considering different mission requirements. For example, a camera with a fixed focal length may require that the variation of the distance from the Earth (and hence the excursion along the $\mathbf{z}$ axis) shall be limited. However, the value of the distance itself is not assigned, as the camera could be mounted with a suitable focal length.

In order to find optimal orbits that have reduced excursion along the $\mathbf{z}$ axis, but still minimise the propellant consumption by settling at the correct altitude, we can devise the following objective function:

$$
J=-m_{f}+\frac{w}{2 \pi} \int_{0}^{2 \pi} v_{z}^{2} \mathrm{~d} t
$$

in which the final mass is weighed together with the integral of the square of the velocity component along the $\mathbf{z}$ axis. Hence, a velocity $v_{z}$ along the orbit is penalising the cost according to the weight $w$. By varying the weight, families of optimal orbits are found, trading off propellant consumption with altitude change throughout the orbit. Table 2 shows, for 5 different values of the weight, the minimum and maximum distance from the Earth, and the respective final mass after one year, assuming an initial mass of $1000 \mathrm{~kg}$ and $\beta_{0}=0.05$. It can be seen that, as the weight increases, the orbit converges to a "flat" orbit parallel to the $\mathbf{x}-\mathbf{y}$

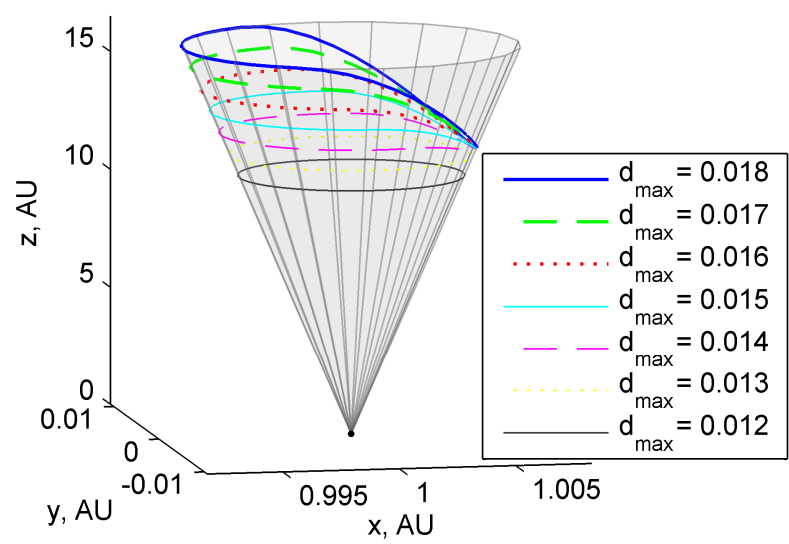

Fig. 8: Optimal orbits obtained constraining the maximum distance from the Earth, for $\beta_{0}=0.05$.

Table 2: Characteristics of optimal orbits obtained minimising a weighed sum of propellant mass and velocity in $\mathbf{z}$, for $\beta_{0}=0.05$.

\begin{tabular}{cccc}
\hline$w$ & $d_{\min }, \mathrm{AU}$ & $d_{\max }, \mathrm{AU}$ & $m_{f}, \mathrm{~kg}$ \\
\hline $5 \cdot 10^{-15}$ & 0.014744 & 0.019960 & 899.638660 \\
$5 \cdot 10^{-14}$ & 0.016982 & 0.017942 & 894.916402 \\
$1 \cdot 10^{-13}$ & 0.017262 & 0.017763 & 894.270074 \\
$2 \cdot 10^{-13}$ & 0.017411 & 0.017668 & 893.914791 \\
\hline
\end{tabular}

plane, at a distance of about 0.017 AU. This is the minimum consumption altitude for a constant-distance orbit [20]. The propellant cost of having a flat orbit with respect to a free one can be quantified as about $8 \mathrm{~kg}$ in the first year for $\beta_{0}=0.05$ (compare Table 1 and Table 2).

Another family of orbits can be found assuming that the payload cannot get further from the Earth than a defined distance. In this case, optimal orbits can be found by using the same objective function as in Eq. (4), but adding the additional state bound:

$$
0 \leq r_{z} \leq d_{\max } \cos \left(\delta_{e q}\right)
$$

This leads to the family of orbits plotted in Fig. 8; Table 3 describes some of their characteristics. Once again limiting the altitude is counterbalanced by additional propellant consumption. We refer to [20] for a more detailed discussion of the different types of polesitter orbits. 
Table 3: Characteristics of optimal orbits with constrained maximum distance, for $\beta_{0}=0.05$.

\begin{tabular}{ccc}
\hline$d_{\max }, \mathrm{AU}$ & $d_{\min }, \mathrm{AU}$ & $m_{f}, \mathrm{~kg}$ \\
\hline 0.018 & 0.013179 & 898.507259 \\
0.017 & 0.013196 & 896.688888 \\
0.016 & 0.013201 & 894.203213 \\
0.015 & 0.013183 & 890.818185 \\
0.014 & 0.013105 & 886.186387 \\
0.013 & 0.012838 & 879.749327 \\
0.012 & 0.012000 & 870.546037 \\
\hline
\end{tabular}

\section{SPACECRAFT FEEDBACK CONTROL}

Due to the instability of pole-sitter orbits (which was verified numerically), a feedback control is necessary to keep the spacecraft on track, counterbalancing errors and small perturbations that are not considered in the dynamics for the reference solution, as well as errors in the spacecraft model (e.g. degradation and nonidealness of the sail).

In this section, a linear quadratic regulator (LQR) will be designed with this aim [27]. It is important to underline that we would like to control the spacecraft using only the thruster; the reason of this choice is twofold: first, the thruster offers three independent control variables, and the thrust vector can in principle be oriented in any direction and have an arbitrary magnitude (this does not happen with the sail force): this guarantees the controllability of the system; second, we assume that steering the thruster or varying the thrust magnitude is cheaper and quicker (in terms of angular momentum change) than changing the attitude of the solar sail. Since the dynamics of the orbit are relatively slow, we neglect the response time of the actuator when designing the feedback control. Also, we assume that the spacecraft has perfect knowledge of its position in space at any time. This can be achieved with a proper tracking system from Earth.

The feedback control is designed as an additional control component which sums to the reference (or feedforward) control. The control system is implemented using MATLAB/Simulink (Fig. 9).

In this paper, a superscripted tilde $(\sim)$ will denote the reference values, as given by the trajectory optimisation. The reference control is transformed into cone/clock angles, such that the control vector over time becomes:

$$
\tilde{\mathbf{u}}(t)=\left[\begin{array}{lllll}
\alpha & \delta & \alpha_{T} & \delta_{T} & T
\end{array}\right]^{T}
$$

where $\alpha_{T}, \delta_{T}$ are the clock and cone angles of the thrust vector $\mathbf{T}$, defined in an analogous way as for $\hat{\mathbf{n}}$. This is done to reduce the control vector to the minimum number of variables and avoid the additional constraint, with respect to the Cartesian version. The reference control is fed into the dynamics of the real system, after passing though a saturation block, which enforces the following control bounds:

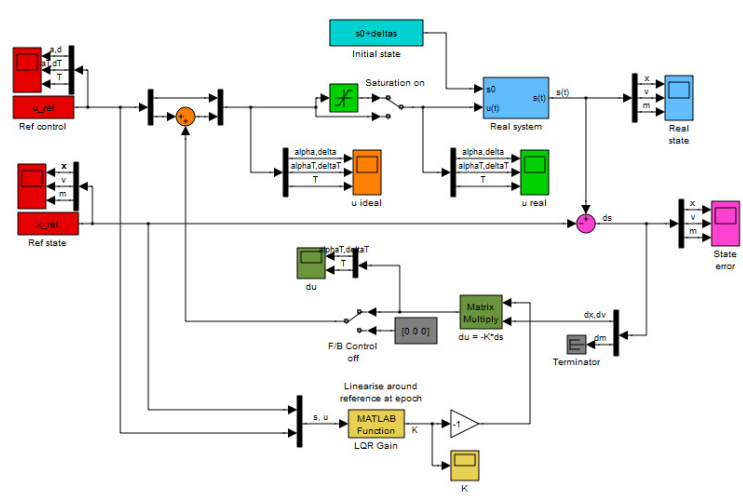

Fig. 9: Simulink block scheme for the feedback control loop.

$$
\begin{aligned}
& \mathbf{u}_{l}=\left[\begin{array}{lllll}
0 & -\infty & -\infty & -\infty & 0
\end{array}\right]^{T} \\
& \mathbf{u}_{u}=\left[\begin{array}{lllll}
\pi / 2 & +\infty & +\infty & +\infty & T_{\max }
\end{array}\right]^{T}
\end{aligned}
$$

For the feedback control loop, the mass state is completely neglected (there is no necessity to follow the reference mass), therefore the reference state vector is simply $\tilde{\mathbf{x}}(t)=[\mathbf{r}, \mathbf{v}]^{T}$.

The control shall be designed such as to follow a time-varying reference in a non-linear system. Therefore, in principle, the gain matrix shall be timevariant and designing using adaptive control theory. However, if we assume that the dynamics of the reference trajectory is slow enough, then we can approximate the time varying problem as a sequence of time-invariant problems, and use classic linear feedback control theory for computing the gain matrix. However, the optimal control problem shall be solved at each instant of time, and the gain matrix updated. The disadvantage of such a control method is that the gain matrix shall be computed in real-time, as a function of the current spacecraft state, with computational load on the spacecraft, and cannot be computed offline.

We consider a generic instant of time $\bar{t}$ : from the trajectory design, it is known that at that time the spacecraft shall be at reference state $\overline{\mathbf{x}}=\tilde{\mathbf{x}}(\bar{t})$ and with reference control $\overline{\mathbf{u}}=\tilde{\mathbf{u}}(\bar{t})$. However, in general, the spacecraft will be at a state $\mathbf{x}$ with control $\mathbf{u}$. If we assume that the real state and control are not distant from the reference state and control, the dynamics of the CR3BP with control can be linearised around the reference state and control, in the following way:

$$
\mathbf{A}_{6 \times 6}(\overline{\mathbf{x}}, \overline{\mathbf{u}})=\left.\frac{\partial \mathbf{f}}{\partial \mathbf{x}}\right|_{\overline{\mathbf{x}}, \overline{\mathbf{u}}} ; \mathbf{B}_{6 \times 3}(\overline{\mathbf{x}}, \overline{\mathbf{u}})=\left.\frac{\partial \mathbf{f}}{\partial\left[\alpha_{T}, \delta_{T}, T\right]}\right|_{\overline{\mathbf{x}}, \overline{\mathbf{u}}}
$$

where the matrix $\mathbf{B}$ is computed differentiating only with respect to the controls that will be used (those related to the thruster). The derivatives can be computed 
analytically offline starting from the expression of $\mathbf{f}$, and then evaluated at a specific point. However their complete expression is omitted here for conciseness.

Hence the dynamics of the system in the vicinity of $\overline{\mathbf{x}}, \overline{\mathbf{u}}$ can be expressed as:

$$
\delta \dot{\mathbf{x}}=\mathbf{A} \delta \mathbf{x}+\mathbf{B} \delta \mathbf{u}
$$

where $\delta \mathbf{x}=\mathbf{x}-\overline{\mathbf{x}}$ and $\delta \mathbf{u}=\left[\alpha_{T}, \delta_{T}, T\right]^{T}-\left[\bar{\alpha}_{T}, \bar{\delta}_{T}, \bar{T}\right]^{T}$ represent the error with respect to the reference condition (see Fig. 10).

In order for the control to be valid, the spacecraft shall be in the vicinity of the reference at any time (i.e. linear approximation).

Within the linear dynamics, the problem is that of following a linearised time-varying reference trajectory, subject to the (linearised) dynamics given by Eq. (5). It can be proven that if the reference follows linear dynamics, then the optimal gain matrix is the same as in the case of a system pursuing $\delta \mathbf{x}=0$, except that this time the transient is on the error with respect to the linearised dynamics of the reference, rather than on $\delta \mathbf{x}$. However, in this case, since the linearisation is done around the reference, and it is updated at every instant of time, it results that the reference state corresponds to $\delta \mathbf{x}=0$ at any time. Therefore, pursuing null error is equivalent to $\delta \mathbf{x}=0$. This can also be explained considering that the feedback control is responsible for tackling small excursions off the reference path, while the reference control is responsible for ensuring that the spacecraft follows the reference states.

The optimal control problem to solve is the one of minimising the quadratic cost function:

$$
J(\delta \mathbf{u})=\int_{0}^{\infty}\left(\delta \mathbf{x}^{T} \mathbf{Q} \delta \mathbf{x}+\delta \mathbf{u}^{T} \mathbf{R} \delta \mathbf{u}+2 \delta \mathbf{x}^{T} \mathbf{N} \delta \mathbf{u}\right) d t
$$

which aims at minimising the state error and the controls over an infinite amount of time, subject to the dynamics in Eq. (5). The matrices $\mathbf{Q}, \mathbf{R}, \mathbf{N}$ are weights that quantify the relative cost of each state and control in the cost function. For this problem, considering the normalisation of the variables, by trial and error they were set to:

$$
\begin{aligned}
& \mathbf{Q}=1000 \cdot \mathbf{I}_{6 \times 6} \\
& \mathbf{R}=\operatorname{diag}\left(\left[\begin{array}{lll}
1 & 1 & 10^{33}
\end{array}\right]\right) \\
& \mathbf{N}=\mathbf{0}_{6 \times 3}
\end{aligned}
$$

The large number in $\mathbf{R}$ can be explained by the fact that the unit of force is small in the non-dimensional system $\left(1 \mathrm{~N}\right.$ is of the order of $\left.10^{-18}\right)$.

Minimising Eq. (6) under the assumption of a control proportional to $\mathbf{e}$, as $\delta \mathbf{u}=-\mathbf{K} \delta \mathbf{x}$, leads to the well known algebraic Riccati equation [27], which can be solved to compute the gain matrix $\mathbf{K}_{3 \times 6}$ for $\mathbf{A}, \mathbf{B}$.

This control is added to the reference, to find the total control to be applied:

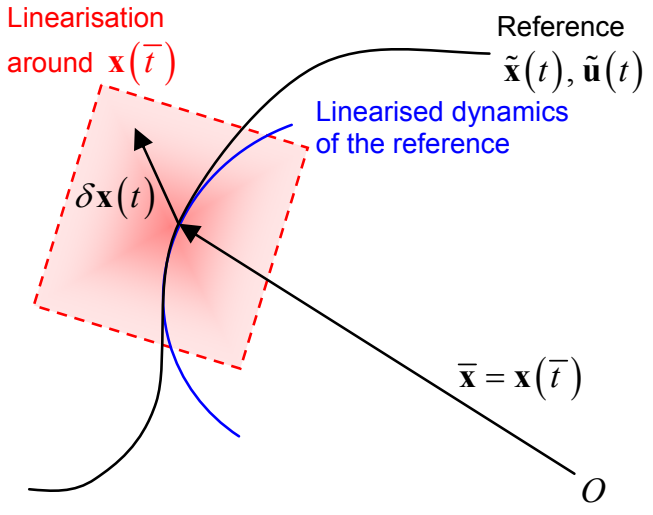

Fig. 10: Linearisation around the reference.
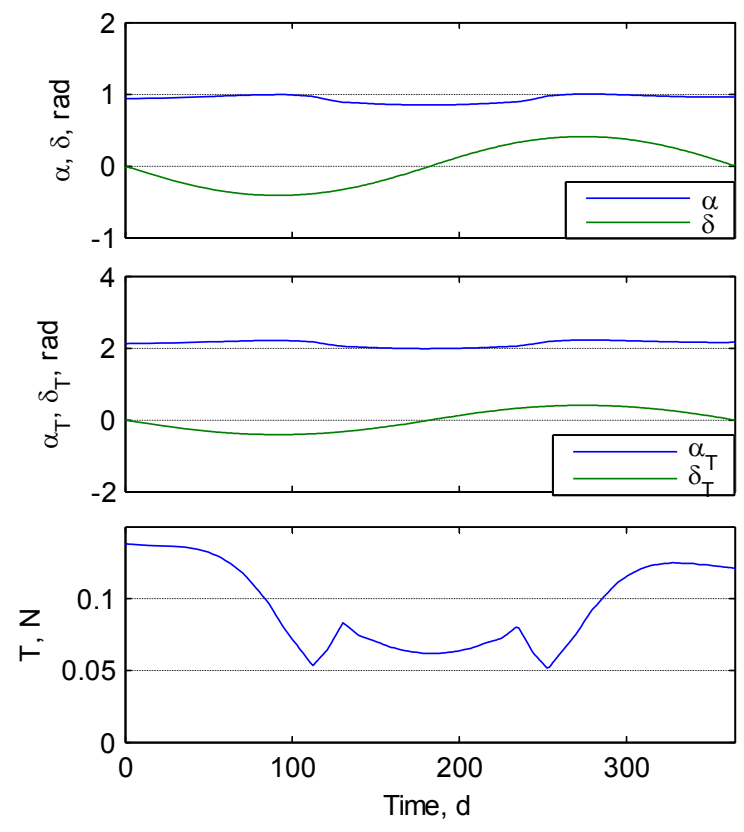

Fig. 11: Reference control for selected orbit.

$$
\mathbf{u}=\tilde{\mathbf{u}}+\left[\begin{array}{lll}
0 & 0 & \delta \mathbf{u}
\end{array}\right]^{T}
$$

to force the spacecraft to follow the reference states.

\section{IV.I. Test cases}

We consider a North Pole observation mission in which the maximum distance of the payload from the Earth is constrained to $d_{\max }=0.018 \mathrm{AU}$. The spacecraft mass at injection into the orbit is $m_{0}=1000 \mathrm{~kg}$ and the lightness number is $\beta_{0}=0.05$, corresponding to a sail size of about $180 \mathrm{~m} \times 180 \mathrm{~m}$. The specific impulse of the thruster is $3200 \mathrm{~s}$.

The optimal orbit for this spacecraft, which will be used as a reference, is represented in black in the following figures (see for example Fig. 12), and its reference control is in Fig. 11, split into angular 
components and thrust magnitude. The maximum thrust available from the SEP system is set to $T_{\max }=0.15 \mathrm{~N}$. It was noted that the performances are very similar on the different orbits proposed in the preceding sections.

We present three different scenarios in which the feedback control plays a fundamental role to guarantee the success of the mission.

\section{Injection Error}

In this first scenario, we hypothesise an injection error of the spacecraft into the pole-sitter orbit starting point. In other words, we consider a perturbed initial state (in position or velocity) at the winter solstice, and start propagating from that state. The aim is to assess the capability of the feedback control to bring the spacecraft to the reference orbit.

It was found that the control is able to recover the spacecraft within $20,000 \mathrm{~km}$ and $20 \mathrm{~m} / \mathrm{s}$ from the reference in less than 1 year if the initial displacement is less than $100,000 \mathrm{~km}$ in any direction $(x, y$ or $z$ ), and less than $100 \mathrm{~m} / \mathrm{s}$ in velocity (being more sensitive to variations of $v_{y}$ ).

Fig. 12 and Fig. 13 show respectively the cases of an injection error in position and velocity. In each figure, plot (a) shows the state error $\delta \mathbf{x}$ with respect to the reference. This vector shall be driven to zero by the feedback control loop. Even if the mass state is not followed, it is plotted to show the gain or loss of mass with respect to reference conditions. Plot (b) shows the control history including the feedback. For sake of comparison, the reference control is also plotted in the same graph, as a dotted line. Finally, plot (c) represents the reference trajectory, the actual trajectory of the spacecraft and the trajectory of the spacecraft if no feedback control is used.

The history of the error in the state vector with respect to the reference (a) shows the initial displacement of one component, then a transient period in which all the components are far from the reference, and finally settlement in its neighbourhood.

Comparing thrust magnitude history (b) with the reference, it can be seen that an initial saturation of the thrust is necessary to bring the spacecraft back to the reference state. Also, oscillations around the reference thrust can be seen, even in the vicinity of the reference state, as is common in many feedback control loops.

If the tests are repeated with increasing values of initial displacement (either position or velocity), the control saturates for a longer and longer time at the beginning of the orbit, and eventually even the saturated thrust is not enough to bring the spacecraft on-track and the trajectory diverges indefinitely from the reference.

It is worth noting that an error in the injection does not necessarily translate into additional propellant consumption to recover the reference trajectory.
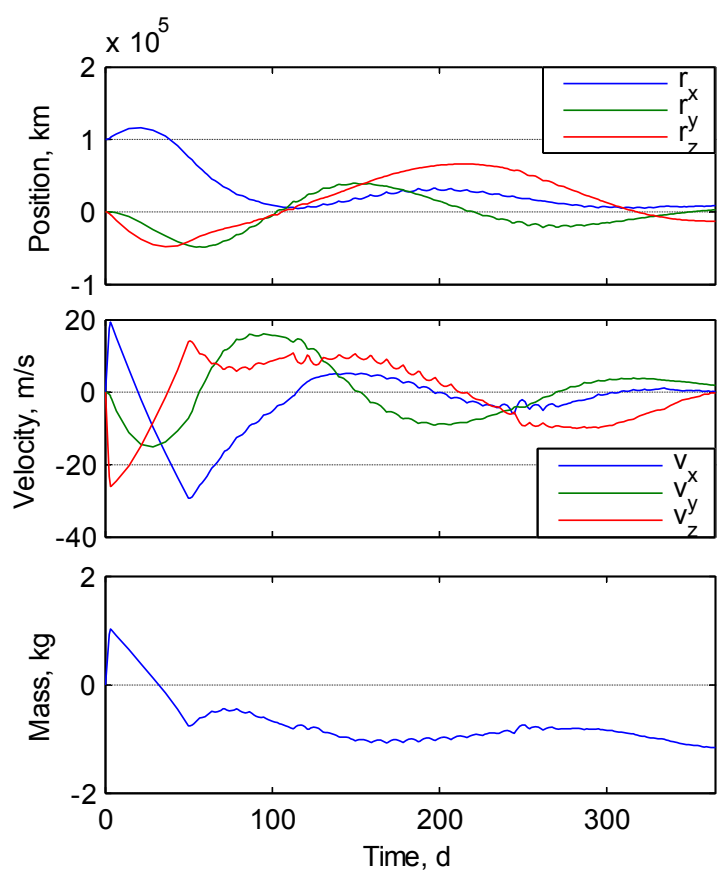

a)
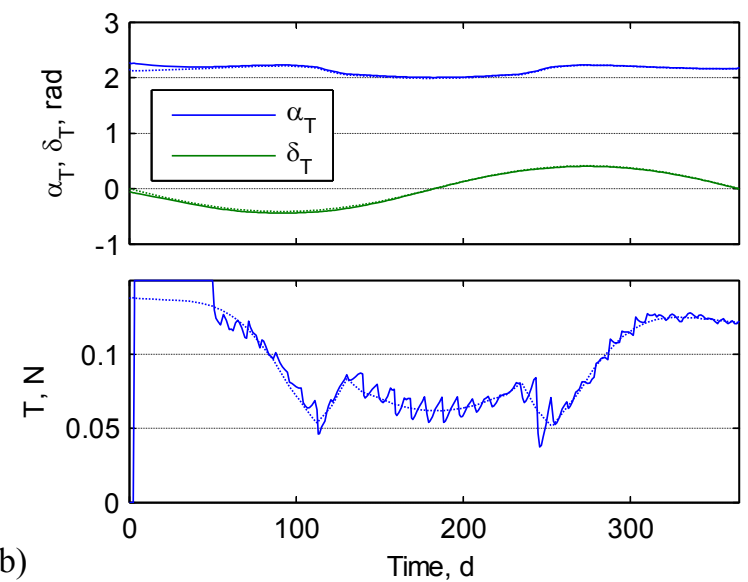

b)
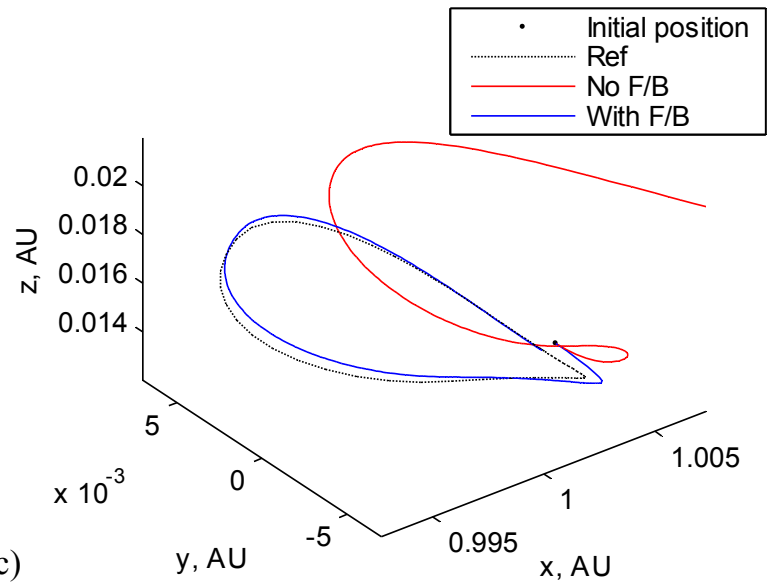

Fig. 12: Initial error of $\Delta r_{x}=100,000 \mathrm{~km}$. (a) State error. (b) Controls (dashed line is the reference). (c) Trajectory (Reference, without control and with control). 

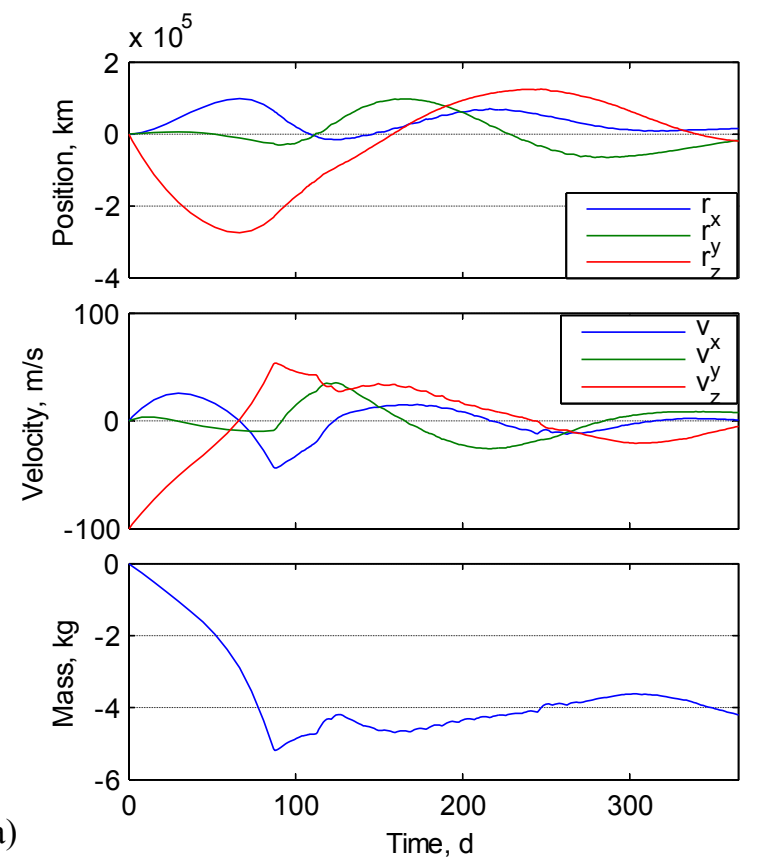

a)
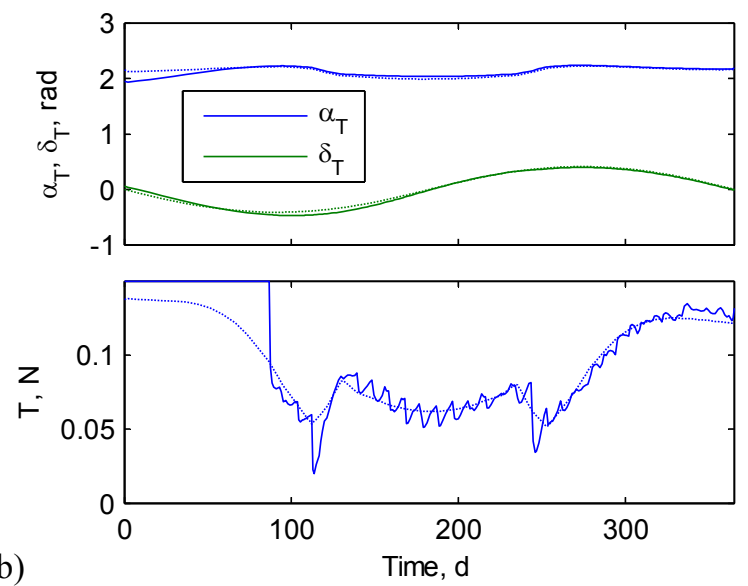

b)

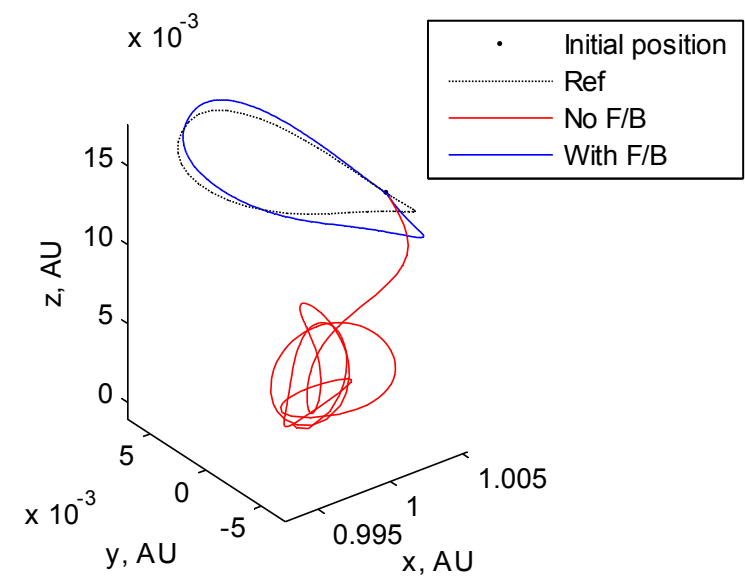

Fig. 13: Initial error of $\Delta v_{z}=-100 \mathrm{~m} / \mathrm{s}$. (a) State error.

(b) Controls (dashed line is the reference). (c) Trajectory (Reference, without control and with control).
However, it always implies a transient trajectory that is not fulfilling the pole-sitter constraint, i.e. the spacecraft is not above the pole.

Finally, the trajectory plot (c) clearly shows that, if no control were used, the spacecraft would not be able to track the reference, but instead would fall into an orbit around the Earth, or escape from it.

\section{$\underline{\text { Sail degradation }}$}

In this case we assume that the spacecraft is injected correctly, but the actual sail reflectivity is reduced to $r_{s}=0.8$. This can happen in case of unexpected wrinkles, or simply due to the degradation of the reflective material after some time.

As it can be seen from Fig. 14, the thruster is able to compensate for the degradation of the sail, keeping the spacecraft in the vicinity of the reference. However, different from the case of error in the injection, the required thrust is constantly above the reference, and this translates into additional propellant consumption. For the considered degradation, the propellant needed is about $7.5 \mathrm{~kg}$ in the first year. This value can be lowered if a new reference optimal trajectory, or at least control law, could be redesigned taking into account the degraded solar sail.

\section{$\underline{\text { SEP failure }}$}

This case involves a sudden, temporary failure of the SEP thruster. Also in this case the feedback control can bring back the spacecraft on-track, provided that the duration of the failure is below a certain limit, which depends on the position along the orbit. Table 4 reports the maximum failure duration in four different positions along the orbit. As expected, the most critical period is winter, in which the part of total acceleration provided by the SEP is highest. Here the SEP cannot be switched off for longer than 20 days, otherwise it would be impossible to recover the mission. In the summer solstice, instead, the failure time can go up to 35 days. These data refer to the first year of the mission. In the following years, the mass of the spacecraft is lower (while the thrust remains the same), and therefore it is expected an increase in these values.

Fig. 15 shows one case of SEP failure. The period of 20 days without thrust is visible in plot (a), and it is followed by about 35 days of full thrust to meet the reference orbit.

Table 4: Maximum SEP failure duration.

\begin{tabular}{cc}
\hline Starting epoch & Maximum duration, d \\
\hline 21 December $(t=0)$ & 20 \\
21 March $(t=\pi / 2)$ & 30 \\
21 June $(t=\pi)$ & 35 \\
21 September $(t=3 \pi / 2)$ & 30 \\
\hline
\end{tabular}




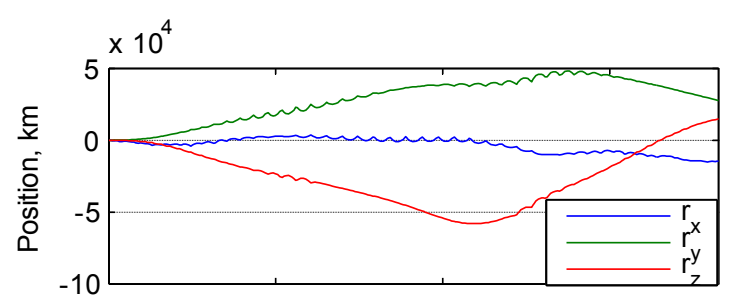

a)
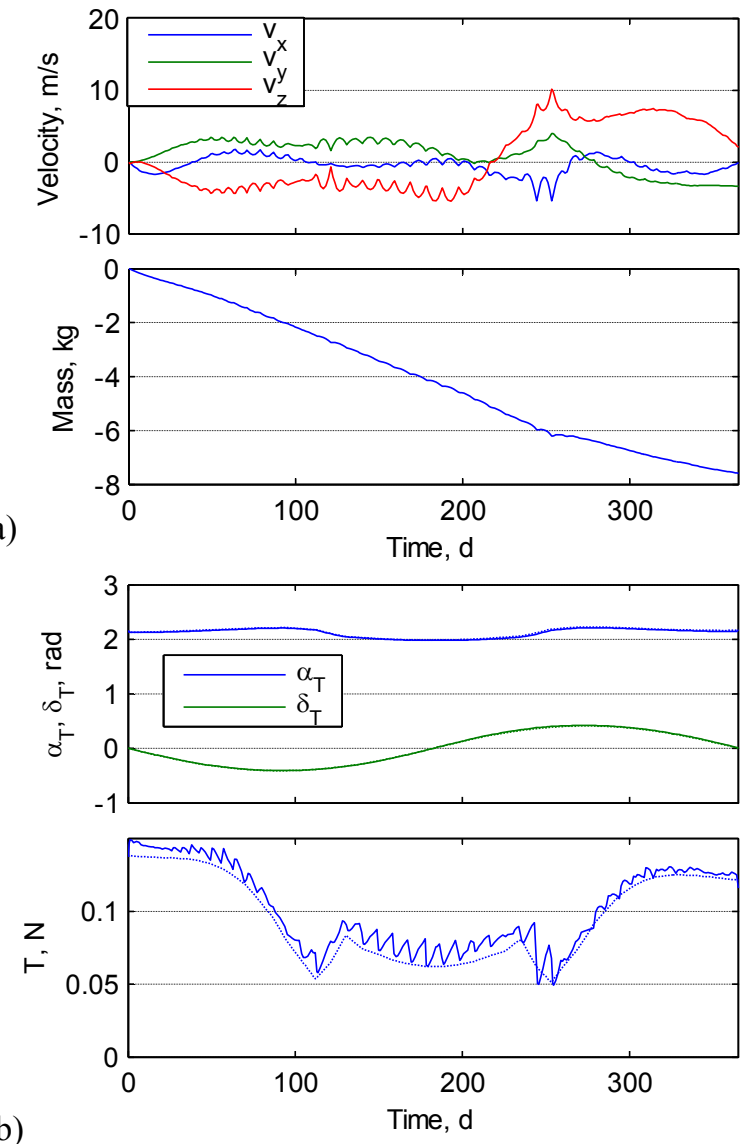

b)

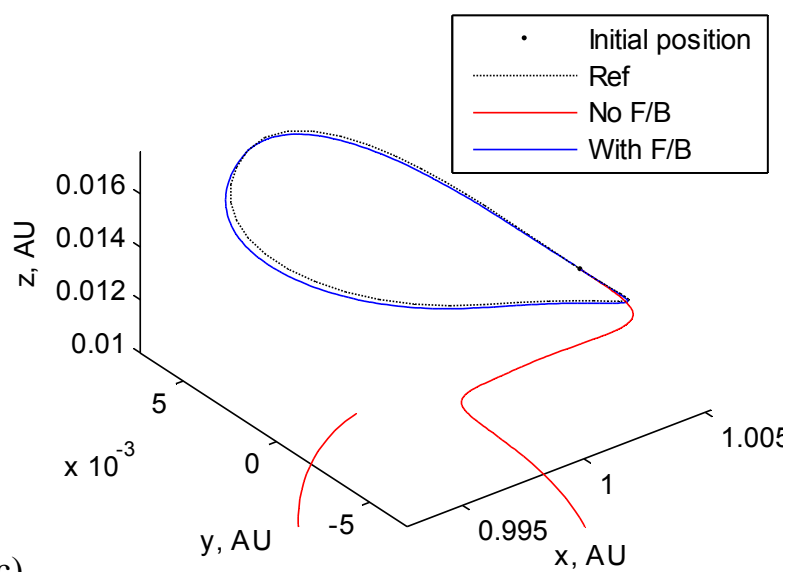

c)

Fig. 14: Sail degradation: reflectivity reduced to $r_{s}=0.8$. (a) State error. (b) Controls (dashed line is the reference). (c) Trajectory (Reference, without control and with control).
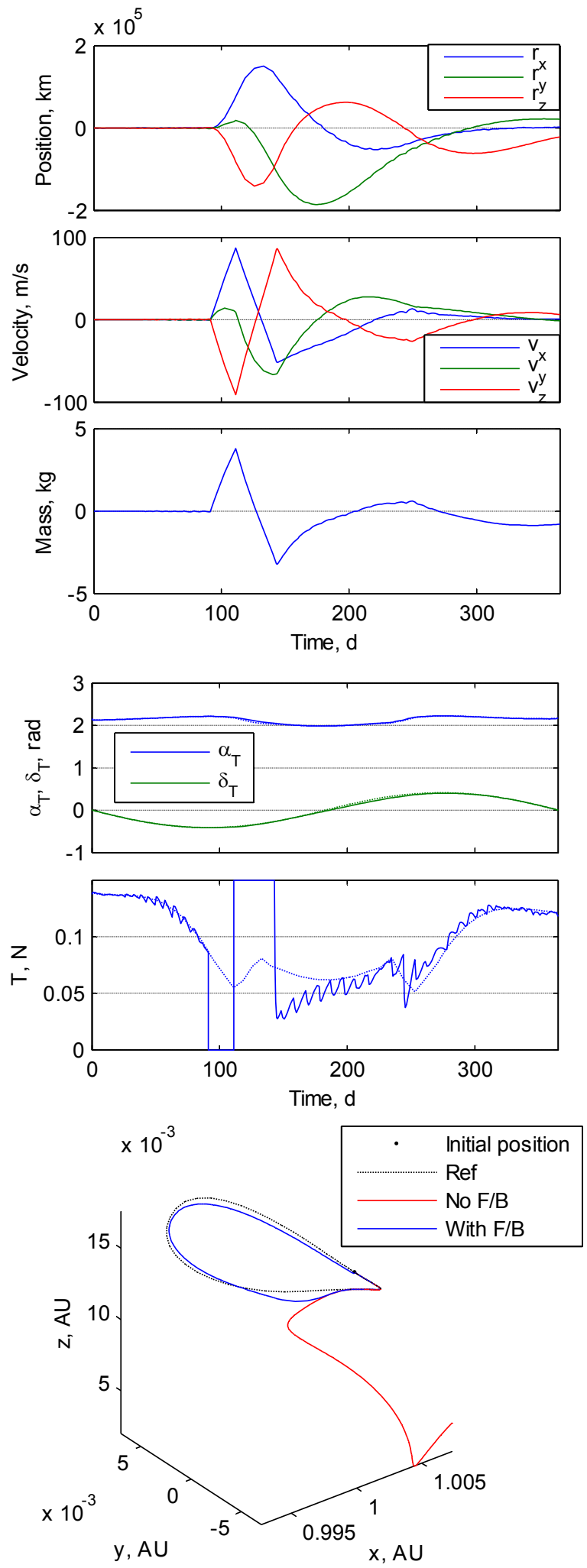

Fig. 15: SEP failure on 21 March $(t=\pi / 2)$, lasting for 20 days. (a) State error. (b) Controls (dashed line is the reference). (c) Trajectory (Reference, without control and with control). 
Note that even a failure of 20 days, as in figure, results in an additional mass consumption of only about $1 \mathrm{~kg}$ (see Fig. 15c). In fact, the mass used to bring the spacecraft on track after the failure is almost completely compensated for by the mass saved during the engine failure. However, as before, during the transient the spacecraft is not precisely above the North Pole.

\section{CONCLUSION}

In this work, the hybridisation of solar electric propulsion (SEP) and solar sailing was proposed for a pole-sitter mission. In addition, a feedback control system was designed to keep the spacecraft on-track under unstable dynamics.

Minimum fuel consumption pole-sitter orbits were presented using hybrid solar electric propulsion (SEP) and solar sail propulsion. A direct method based on pseudo-spectral discretisation was demonstrated to be reliable and suitable for this aim, guaranteeing fast convergence to the optimal solution. The combination of the two propulsion systems allows propellant saving over the pure SEP spacecraft, potentially enabling longer missions; at the same time, the presence of lowthrust propulsion enables orbits that cannot be achieved with a pure sail. The analysis took into account freedistance orbits, as well as orbits that constrain their distance from the Earth to meet possible payload requirements, at the cost of some propellant mass.

The proposed feedback control proved that is possible to maintain the spacecraft in the reference condition, despite the instability of the natural dynamics, only by using the SEP thruster. Moreover, it was shown that very little variation on the reference thrust vector are enough to respond to large injection errors, and relatively long SEP failures. This allows keeping the sail on the reference attitude, and avoiding fast slew manoeuvres of the sail.

\section{ACKNOWLEDGEMENTS}

This work was funded by the European Research Council, as part of project 227571 VISIONSPACE. The authors thank Dr. James Biggs for useful discussions and Dr. Victor Becerra for providing PSOPT.

\section{REFERENCES}

1. J. M. Driver, "Analysis of an arctic polesitter", Journal of Spacecraft and Rockets, vol. 17, n. 3, 1980

2. "CryoSat mission and data description", 2007, http://esamultimedia.esa.int/docs/Cryosat/Mission and Data_Descrip.pdf, Cited: 2 January 2007

3. M. A. Lazzara, "The polar meteorlogist's dream machine: artificial Lagrange orbit satellite applications via arctic and antarctic composite satellite imagery", in Proceedings of $2^{\text {nd }}$
International Symposium on Solar Sailing (ISSS 2010), New York, NY, USA, 2010

4. N. C. Wallace, "Testing of the QinetiQ T6 thruster in support of the ESA BepiColombo Mercury mission", in Proceedings of Space Propulsion 2004 - 4th International Spacecraft Propulsion Conference, June 2, 2004 - June 4, 2004, Sardinia, Italy, 2004

5. C. R. McInnes, "Solar sailing: technology, dynamics and mission applications", SpringerPraxis books in astronautical engineering, ed. J. Mason, Springer-Verlag, Berlin, 1999

6. F. A. Tsander, "From a scientific heritage (Translation of "Iz nauchnogo naslediya")", NASA Technical Translation, NASA, 1969

7. O. Mori, H. Sawada, R. Funase, T. Endo, M. Morimoto, et al., "Development of first solar power sail demonstrator - IKAROS", in Proceedings of 21 st International Symposium on Space Flight Dynamics, ISSFD 2009, Toulouse, France, 2009

8. H. Baoyin, C. R. McInnes, "Solar sail equilibria in the elliptical restricted three-body problem", Journal of Guidance, Control, and Dynamics, vol. 29, n. 3, p. 538-543, 2006

9. G. Mengali, A. A. Quarta, "Solar sail trajectories with piecewise-constant steering laws", Aerospace Science and Technology, vol. 13, n. 8, p. 431-441, 2009

10. G. Mengali, A. A. Quarta, "Optimal threedimensional interplanetary rendezvous using nonideal solar sail", Journal of Guidance, Control, and Dynamics, vol. 28, n. 1, p. 173-177, 2005

11. C. R. McInnes, "Artificial Lagrange points for a partially reflecting flat solar sail", Journal of Guidance, Control, and Dynamics, vol. 22, n. 1, p. 185-187, 1999

12. C. R. McInnes, A. J. McDonald, J. F. L. Simmons, E. W. MacDonald, "Solar sail parking in restricted three-body systems", Journal of Guidance, Control, and Dynamics, vol. 17, n. 2, p. 399-406, 1994

13. J. Simo, C. R. McInnes, "Solar sail orbits at the Earth-Moon libration points", Communications in Nonlinear Science and Numerical Simulation, vol. 14, n. 12, p. 4191-4196, 2009

14. M. Leipold, M. Götz, "Hybrid photonic/electric propulsion", Kayser-Threde GmbH, Munich, Germany, 2002

15. S. Baig, C. R. McInnes, "Artificial three-body equilibria for hybrid low-thrust propulsion", Journal of Guidance, Control, and Dynamics, vol. 31, n. 6, p. 1644-1655, 2008

16. G. Mengali, A. A. Quarta, "Trajectory design with hybrid low-thrust propulsion system", Journal of Guidance, Control, and Dynamics, vol. 30, n. 2, p. 419-426, 2007 
17. J. Simo, C. R. McInnes, "Displaced periodic orbits with low-thrust propulsion", in Proceedings of $19^{\text {th }}$ AAS/AIAA Space Flight Mechanics Meeting, Savannah, Georgia, USA, 2009

18. M. Macdonald, C. R. McInnes, "Solar sail mission applications and future advancement", in Proceedings of $2^{\text {nd }}$ International Symposium on Solar Sailing (ISSS 2010), New York, NY, USA, 2010

19. R. L. Forward, "Statite: a spacecraft that does not orbit", Journal of Spacecraft and Rockets, vol. 28, n. 5, p. 606-611, 1991

20. M. Ceriotti, C. R. McInnes, "An Earth pole-sitter using hybrid propulsion", in Proceedings of AIAA/AAS Astrodynamics Specialist Conference, Toronto, Ontario, Canada, 2010

21. M. Ceriotti, C. R. McInnes, "A near term polesitter using hybrid solar sail propulsion", in Proceedings of $2^{\text {nd }}$ International Symposium on Solar Sailing (ISSS 2010), New York, NY, USA, 2010

22. B. Dachwald, G. Mengali, A. A. Quarta, M. Macdonald, "Parametric model and optimal control of solar sails with optical degradation", Journal of Guidance, Control, and Dynamics, vol. 29, n. 5, p. 1170-1178, 2006

23. V. M. Becerra, "PSOPT", available from: http://www.psopt.org/

24. F. Fahroo, I. M. Ross, "Direct trajectory optimization by a Chebyshev pseudospectral method", Journal of Guidance, Control, and Dynamics, vol. 25, n. Compendex, p. 160-166, 2002

25. G. Elnagar, M. A. Kazemi, M. Razzaghi, "Pseudospectral Legendre method for discretizing optimal control problems", IEEE Transactions on Automatic Control, vol. 40, n. 10, p. 1793-1796, 1995

26. J. Brophy, "Advanced ion propulsion systems for affordable deep-space missions", Acta Astronautica, vol. 52, p. 309-316, 2003

27. A. E. Bryson, Y.-C. Ho, "Applied optimal control: optimization, estimation, and control (Revised printing)", Taylor \& Francis Group, New York, 1975 